\title{
An Overview of Solar Sail Propulsion within NASA
}

\author{
Les Johnson $^{1}$ \\ NASA George C. Marshall Space Flight Center, Huntsville, AL 35812, USA \\ Grover A. Swartzlander ${ }^{2}$ \\ Rochester Institute of Technology, Rochester, NY 14623 \\ and \\ Alexandra Artusio-Glimpse ${ }^{3}$ \\ Rochester Institute of Technology, Rochester, NY 14623
}

\begin{abstract}
Solar Sail Propulsion (SSP) is a high-priority new technology within The National Aeronautics and Space Administration (NASA), and several potential future space missions have been identified that will require SSP. Small and mid-sized technology demonstration missions using solar sails have flown or will soon fly in space. Multiple mission concept studies have been performed to determine the system level SSP requirements for their implementation and, subsequently, to drive the content of relevant technology programs. The status of SSP technology and potential future mission implementation within the United States (US) will be described.
\end{abstract}

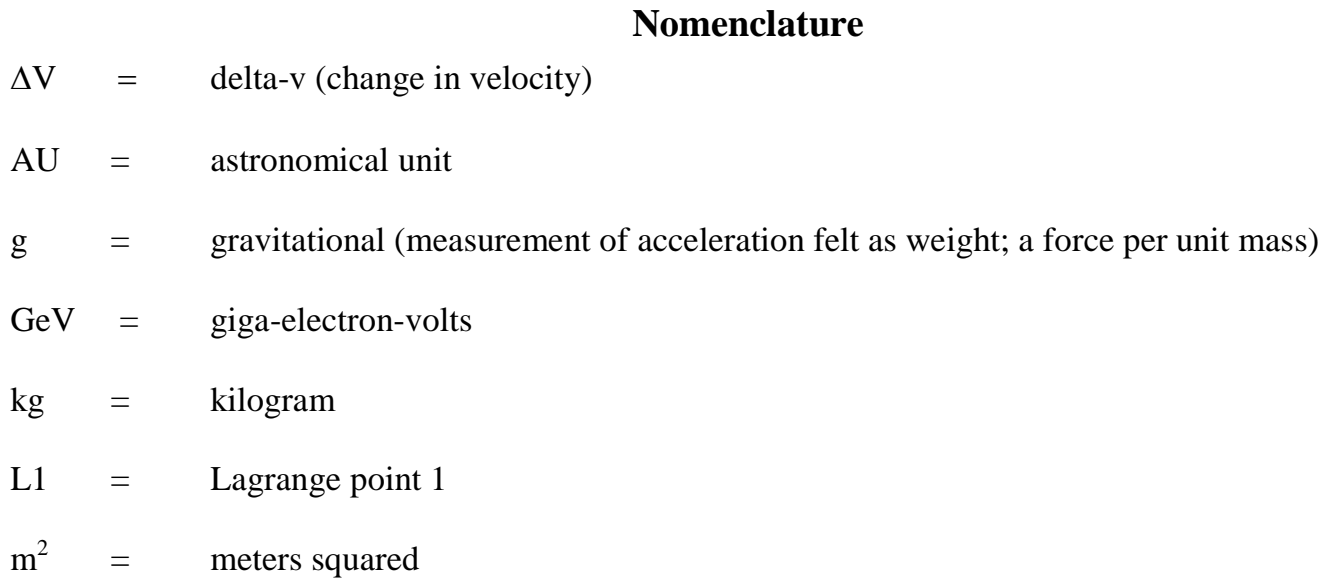

\section{Introduction}

Solar Sail Propulsion (SSP) was identified as a high-priority new technology by the Space Studies Board of the National Research Council in their 2012 Heliophysics Decadal Survey [1], and several potential future space

\footnotetext{
${ }^{1}$ Deputy Manager, Advanced Concepts Office, Mail Code ED04, NASA MSFC, Huntsville, AL 35812

${ }^{2}$ Associate Professor, Department of Physics, 54 Lomb Memorial Drive, Rochester, NY 14623

${ }^{3}$ Graduate Student, Department of Physics, 54 Lomb Memorial Drive, Rochester, NY 14623
} 
missions have been proposed that will require SSP to be achieved. Missions identified include those that would support enhanced space weather monitoring and prediction by placing an instrumented satellite at Sun/Earth L1 (often called Heliostorm), high inclination solar orbiting science satellites (Solar Polar Imager), and an ambitious short-travel time mission to beyond the Heliopause (also known as The Interstellar Probe).

This renewed interest in solar sails is manifested by several small and mid-sized technology demonstration missions within the US that use solar sails, ranging in size from cubesat-scale sails designed for primary operation in Earth orbit to 40-meter class sails capable of operating anywhere in Earth-Sun space. These include the NASA Marshall Space Flight Center's (MSFC) already-flown NanoSail-D, the CubeSail being developed by The University of Illinois at Urban/Champaign, and the L'Garde/NASA Sunjammer.

Several mission concept studies have also been performed to determine the system level SSP requirements for their implementation and, subsequently, to drive the content of NASA's technology programs. NASA's MSFC recently studied the use of SSP in a Mars sample return architecture, providing a potential cost reduction to the overall mission by eliminating the need for one of the three launch vehicles required in the baseline chemical propulsion approach [2]. Another MSFC-led study examined the use of SSP to enable asteroid survey missions to perform low-cost reconnaissance of candidate Near Earth Asteroids (NEAs) for future human exploration [3]. The NASA Innovative Advanced Concepts (NIAC) program is funding innovative advanced sail technology studies, such as the "Steering of Solar Sails Using Optical Lift Force" [4] and "Solar System Escape Architecture for Revolutionary Science (SSEARS)" [5] studies. Various academic studies have been conducted looking at very advanced sails and architectures, including those that make use of new materials like graphene for enabling interstellar missions [6] and flotillas of sails to act as sunshades for mitigating the effects of global warming by reducing the incident solar flux [7].

\section{Solar Sails In Perspective: 1990's to the Present}

While SSP systems have been studied since the early twentieth century, it wasn't until the 1990's that serious engineering development began. In 1993, the Russian Space Agency conducted a successful solar sail experiment, called Znamya, which was deployed from an unmanned Progress vehicle after it departed from their Mir Space Station. A 20-meter circular sail-like reflector was successfully deployed; a follow-up experiment in 1999 collided with a deployed spacecraft antenna and was destroyed [8].

The first free-flying and autonomous solar sail spacecraft built for flight was The Planetary Society's privately funded Cosmos 1. Once launched, the sail was to have deployed eight triangular 'heliogyro' blades in 
LEO and demonstrated controllable solar sailing [9]. Unfortunately, the sailcraft never reached orbit due to a launch vehicle failure.

In 1999, Germany's Deutschen Zentrum fur Luft-und Raumfahrt (DLR) performed a series of ground system demonstration tests of a 20-meter 3-axis stabilized solar sail system. The system was not flown in space, but it clearly showed that the technology was mature enough to be considered for near-term space mission applications [10].

Partially in response to these significant international milestones, in the early 2000's NASA made substantial progress in the development of its own solar sail propulsion systems. Two different 20-meter solar sail systems were produced. The two design configurations successfully completed functional vacuum testing in the Glenn Research Center's Space Power Facility at Plum Brook Station, Ohio. The sails were designed and developed by Alliant Techsystems Space Systems and L'Garde, respectively. Life and space environmental effects testing of sail and component materials were also conducted [11]. NASA planned to fly a 40-meter class solar sail in 1999 , but the project was canceled due to funding constraints.

JAXA began a series of deployment test flights in 2004, leading to the successful flight of the Interplanetary Kite-craft Accelerated by Radiation Of the Sun (IKAROS) in 2010. The IKAROS is the first deep-space demonstration of solar sailing. The IKAROS verified solar radiation pressure effects on the sail and performing in-flight guidance and navigation techniques using the solar sail [12].

Using sail material left over from its ground system demonstrations, NASA MSFC developed NanoSail-D, a cubesat-based solar sail deployment verification experiment. It was not intended for actual solar sailing, but rather as a demonstration of a sail to act as an atmospheric drag device for deorbit. The initial launch failed in 2008. A second attempt was made in 2010 using the flight spare. The second launch attempt was succeeded, the sail deployed, and the spacecraft reentered the atmosphere several months later [13].

\section{Potential Future Missions and Concepts}

As SSP technology matures, entirely new classes of high $\Delta \mathrm{V}$ deep space missions will be enabled. Many of the scientific questions these missions address have been unanswered for decades because the technology to fly spacecraft to the destinations required to address them was not in reach. With the successes of NanoSail-D and IKAROS, that is no longer the case, and the scientific community is starting to seriously plan for using SSPpropelled spacecraft to answer them.

\section{A. Solar Polar Imager}


Taking advantage of a solar sail's ability to provide nearly unlimited total thrust, an SSP spacecraft can fly into a highly-inclined orbit around the Sun in order to study the Sun's poles. Current spacecraft observations of the Sun are limited to being in or very nearly in the ecliptic plane, limiting solar observations to the solar equator or, at best, mid-latitude regions. A 150-meter class solar sail could slowly raise its inclination from the ecliptic plane and reach a solar polar orbit within just a few years, easily reaching an orientation only previously possible with multiple gravity assist maneuvers and considerable flight time [14].

\section{B. Heliostorm}

Space between the Earth and the Sun is not empty. Events on the Sun, such as Coronal Mass Ejections (CMEs) send massive clouds of plasma into space with energies as high as $1 \mathrm{GeV}$. The radiation in these plasma clouds is significant and can cause damage to unprotected spacecraft and humans operating beyond the protection of the Earth's magnetosphere. The events that occur in this ever-changing space environment are called "space weather."

The Heliostorm mission would use a solar-sail propelled spacecraft to station a space weather detection and characterization payload sunwards of the classical L1 point along to the Sun-Earth line, potentially placing it twice as close to the sun's natural L1 point at $0.993 \mathrm{AU}$. The sail is used to maintain an artificial equilibrium position displaced both sunward of and slightly off the Sun-Earth line. Onboard instruments will detect solar CMEs much earlier than is now possible and potentially double the warning time of impending terrestrial space weather events [15]

\section{Interstellar Probe}

Humanity's first intentional step into nearby interstellar space may occur with the launch of the Interstellar Probe (ISP) [16]. The ISP would use a $160,000 \mathrm{~m}^{2}$ solar sail with an areal density of less than $1 \mathrm{~g} / \mathrm{m}^{2}$ to achieve a flight time of $20-25$ years to $250 \mathrm{AU}$. The velocity required for such a short flight time would be achieved by having the sail make a very close solar flyby, taking advantage of the increased solar flux and the resultant increased solar photon pressure, to accelerate the sailcraft. The ISP would be instrumented to study the heliopause and the interstellar medium and make a significant penetration into nearby interstellar space, with a minimum goal of reaching $250 \mathrm{AU}$ and a desire to reach as far as $400 \mathrm{AU}$ before the spacecraft systems cease functioning. Sails this large are significantly beyond the state-of-the-art, and additional research and development will be required in sail materials, deployment, and attitude control systems to make it viable. 
Additional missions to ever-increasing interstellar distances would follow, culminating in one traveling to about 1000 AU, aptly named the Thousand AU, (TAU) Mission.

\section{Near Earth Asteroid Rendezvous}

Crewed missions to NEAs are planned for early in the 2020's. To reduce the inherent risk associated with visiting any solar system body for the first time, an assessment of it must be performed robotically, so the crew systems appropriate for the NEAs unique environment can be developed. For example, operations on or near an asteroid composed of a single large piece of rock will be very different from those required to support the exploration of a loosely gravitationally-bound "rubble pile" asteroid.

Conventional rockets, and even unconventional ones using solar electric propulsion, have been considered for this class of precursor mission, and none can match the ability of a solar sail to visit multiple targets sequentially in a relatively short period of time.

NASA MSFC studied various options for implementing an SSP NEA precursor mission and found that an 80-meter 3-axis stabilized solar sail spacecraft launched by a small rocket can easily rendezvous with asteroids 1999 AO10, Apophis, and 2001 QJ142 - all of which are on the most recent listing of "Human Accessible NEAs" - within six years of launch. The notional spacecraft science payload was selected to be simple and lightweight, consisting of only the multispectral imager flown on the NEAR Mission to 433 Eros and 253 Mathilde. Most non-sail spacecraft systems baselined were used in the Messenger and other flight-proven mission spacecraft.

\section{E. Mars Sample Return}

Scenarios for robotic missions to return samples from other planets are inherently complex and will require the sequential launch of many spacecraft, landers, and sample return stages to implement. For this type of mission, as well as any multi-staged space system, it is clear that by minimizing the mass of the final stage, the initial system mass will be reduced by simply reducing the propellant required in earlier stages. Therefore, the best way to minimize the launch mass of the Mars Sample Return (MSR) mission is to reduce the mass of the Earth return stage (ERS).

Using chemical or even solar electric propulsion, the Mars ERS will require a significant amount of fuel to leave Mars orbit and travel back to Earth; hence, the earlier mission stages will be required to transport this mass to Mars, in turn requiring more propellant in these mission stages. In fact, under current designs, nearly half of the orbiter mass is dedicated to returning samples to Earth and stopping the Earth-return propellant at Mars. By 
replacing the chemical propulsion system of the ERS with a solar sail, most, if not all, of this fuel is eliminated. This reduces the fuel mass of the earlier stages and can result in smaller spacecraft, fewer required launches, or both.

The current MSR orbiter is planned to arrive two years ahead of the Mars ascent vehicle (MAV) and lander. The orbiter will provide communication links to the lander and monitor the lander's entry, descent, and lander phases. The orbiter is also required to be the active partner in the rendezvous and capture of the sample canister once the MAV launches it from the surface of Mars.

NASA MSFC studied the use of a solar sail to perform the Earth return portion of the MSR Mission. All other required propulsive functions would still be performed with traditional storable liquid propellant thrusters. Upon retrieval of the sample canister, the solar sail will deploy and begin the low-thrust spiral out of the Martian gravity well. As a means to save mass and reduce the required size of the solar sail, it is proposed that the liquid propulsion system be jettisoned in Mars orbit, potentially reducing the mass of the return system by an additional 10-20 percent.

MSFC also studied the use of a solar sail to perform the Earth-Mars transfer, as well as the return leg. While this would entirely eliminate the orbiter propulsion system and further reduce the system's mass, it would increase the overall trip time beyond what is required for the all-chemical mission approach. It also may be possible, given the large surface area of a solar sail, to use the sail to perform an aerocapture maneuver through the extremely thin upper atmosphere of Mars. .

\section{Technology Development and Demonstrations}

Within NASA, solar sail propulsion is moving forward to mission implementation on both the large $(100 \mathrm{~kg}$ class spacecraft payloads) and small (cubesat) scales. From Sunjammer's planned deep space flight validation to the Earth-orbital CubeSail, solar sail capability is advancing rapidly.

\section{A. Sunjammer}

The L'Garde Sunjammer Mission will demonstrate large solar sails ( 1200 $\left.\mathrm{m}^{2}\right)$ for future NASA mission use. The Sunjammer design relies on L'Garde inflatable rigidizable technology for the sail's structure. The sail structure consists of four conically-stowed, deployable, cold-rigidized booms. These four booms are connected at the center of the sail area to the sailcraft bus. Shortly after launch, the solar sail will be deployed to full size and separated from the carrier. Within a few days of launch, the sail will be calibrated and trimmed, and for the next several weeks, the sail will demonstrate attitude control, guidance, navigation, and control by flying in deep 
space to near the Earth-Sun L1 and finally to sub-L1. Along the way, an onboard magnetometer will be measuring magnetic field and comparing the results with The National Oceanic and Atmospheric administration (NOAA's) Advanced Composition Explorer. It is hoped that these measurements will foster infusion of this technology into the space weather monitoring community. Launch is anticipated in 2014 [17].

\section{B. Steering of Solar Sails Using Optical Lift Force}

Scientists at the Rochester Institute of Technology (RIT) found that transparent refractive objects may settle into a position where they feel a force that is perpendicular to the incoming light direction. Because this transverse force is analogous to the lift experience by an airplane wing, it is called "optical lift." Dr. Swartzlander and his team at RIT completed a NIAC Phase I study in 2001 looking at the physics, technology, and mission applications of optical lift with an emphasis on their application to solar sailing.

Optical wing structures were theoretically and numerically analyzed, and prototype arrays of wings called optical flying carpets, were fabricated with solar sail material clear polyimide (CP1). Various optical wing sizes and shapes were analyzed to develop design strategies for thrust and torque applications. The developed raytracing model has undergone continual advancement and stands as an effective tool for modeling most types of solar sails.

The distributed forces and torques have been reduced to a simple theoretical, whereby the fundamental mechanics may be understood in terms of the numerically-determined center of pressure offset from the center of mass. This research has established a foundation for understanding the force and torque afforded by optical wings. The study began by considering transparent wings and ended by considering wings having a reflecting face. The latter was found to afford the advantages of high thrust and both intrinsic and extrinsic torque. The discovery of the intrinsic torque on optical wings (meaning that a moment arm is not required) has no analogy for a flat reflective solar sail, and therefore provides an extra degree of control that may be useful for sailcraft attitude and navigation purposes.

\section{Solar System Escape Architecture for Revolutionary Science (SSEARS)}

NASA's Jet Propulsion Laboratory (JPL) is exploring the feasibility of using 10 or more sail-propelled probes to explore the heliopause in multiple orthogonal locations in an integrated and synergistic approach, providing a three dimensional map of the solar system's boundaries. 


\section{Conclusion}

For many years, solar sail propulsion was an interesting idea with many mission benefits, but within NASA, the technology was without mission pull. In other words, no official future missions were being planned that would require their use. Since the success of IKAROS and the selection and funding of the Sunjammer demonstration mission, the reality of solar sails has manifested in the Agency's planning, and there are now numerous mission and project teams considering their use.

\section{References}

[1] National Research Council, Solar and Space Physics: A Science for a Technological Society, Washington, DC: The National Academies Press, 2012.

[2] Johnson, L., Using A Solar Sail to Significantly Reduce Total MSR Launch Mass, Concepts and Approaches for Mars Exploration, 12-14 June, 2012, Houston, Texas, USA

[3] Johnson, L.; Alexander, L.; Fabisinski, L.; Heaton, A.; Miernik, J.; Stough, R.; Wright, R.; Young, R., Multiple NEO Rendezvous Using Solar Sail Propulsion, Global Space Exploration Conference; 22-24 May 2012; Washington, DC, USA

[4] Artusio-Glimpse, A., Peterson, T. and Swartzlander, G., Refractive optical wing oscillators with one reflective surface, Opt. Lett. 38, 935-937 (2013)

[5] Nosanov,J., Grebow, D., Trease, B., West, J., and Garrett, H., Solar System Escape Architecture for Revolutionary Science, NASA NIAC Conference, 2013

[6] Matloff, G., Graphene, the Ultimate Interstellar Solar Sail Material?, Journal of the British Interplanetary Society, vol. 65 , p. $378-381$

[7] Kennedy, R., Roy, K., and Fields, D., Dyson Dots: Changing the solar constant to a variable with photovoltaic lightsails, Acta Astronautica, Volume 82, Issue 2, February 2013, Pages 225-237

[8] Leipold, M., Garner,C., Freeland, R., Hermann, A., Noca, M., Pagel, G., Seboldt, W., Sprague, G., and Unckenbold, W., ODISSEE - A proposal for demonstration of a solar sail in earth orbit, Acta Astronautica Volume 45, Issues 4-9, August-November 1999, Pages 557-566

[9] Friedman, L., Pichkhadze, K., Kudryashov, V., Rogovsky, G., Linkin, V., Gotlib, V., Lipatov, A., Cantrell, J., and Garvey, J., COSMOS 1: the Attempt to Fly the First Solar Sail Mission, IAF abstracts, 34th COSPAR Scientific Assembly, The Second World Space Congress, held 10-19 October, 2002 in Houston, TX, USA., p.IAA-11-1-03

[10] Leipold, M., Eiden, M., Garner, C., Herbeck, L., Kassing, D., Niederstadt, T., Krüger, T., Pagel, G., Rezazad, M., Rozemeijer, H., Seboldt, W., Schöppinger, C., Sickinger, S., and Unckenbold, W., Solar sail technology development and demonstration, Acta Astronautica, Volume 52, Issues 2-6, January-March 2003, Pages 317-326 
[11] Johnson, L., Young, R., and Montgomery, E., Recent advances in solar sail propulsion systems at NASA, Acta Astronautica, Volume 61, Issues 1-6, June-August 2007, Pages 376-382

[12] Tsuda, Y., Mori, O., Funase, R., Sawada, H., Yamamoto, H., Saiki, T, Endo, T., and Kawaguchi, J., Flight status of IKAROS deep space solar sail demonstrator, Acta Astronautica, Volume 69, Issues 9-10, November-December 2011, Pages 833-840

[13] Johnson, L., Whorton, M., Heaton, A., Pinson, R., and Laue, G., NanoSail-D: A solar sail demonstration mission, Acta Astronautica, Volume 68, Issues 5-6, March-April 2011, Pages 571-575

[14] Dachwald, B., Ohndorf, A., and Wie, B., Solar Sail Trajectory Optimization for the Solar Polar Imager (SPI) Mission, AIAA/AAS Astrodynamics Specialist Conference and Exhibit, 21 - 24 August 2006, Keystone, CO, USA

[15]Young, R., Updated Heliostorm Warning Mission: Enhancements Based on New Technology, 48th AIAA/ASME/ASCE/AHS/ASC Structures, Structural Dynamics, and Materials Conference, 23-26 April 2007, Honolulu, HI, USA

[16] Liewer, P., Mewaldt, R., Ayon, J., Garner, C., Gavit, S., and Wallace, R., Interstellar Probe using a Solar Sail: Conceptual Design and Technological Challenges, COSPAR Colloquia Series, Volume 11, 2001, Pages 411-420, The Outer Heliosphere - The Next Frontiers

[17] Johnson, L., Young, R., Barnes, N., Friedman, L., Lappas, V., and McInnes, C., Solar Sails: Technology and Demonstration Status, Int'1 J. of Aeronautical \& Space Sci. 13(4), 267-281 (2012) 